\title{
ACSL4 contributes to sevoflurane-induced ferroptotic neuronal death in SH-SY5Y cells via the 5' AMP-activated protein kinase/ mammalian target of rapamycin pathway
}

\author{
Lei Cheng ${ }^{1}$, Xiaodan Zhu' ${ }^{2}$ Yang Liu ${ }^{1}$, Kai Zhu ${ }^{3}$, Kang Lin ${ }^{4}$, Fujun Li $^{1}$ \\ ${ }^{1}$ Department of Anesthesiology, The First Affiliated Hospital of Harbin Medical University, Harbin, China; ${ }^{2}$ Department of Endocrinology, The \\ First Affiliated Hospital of Harbin Medical University, Harbin, China; ${ }^{3}$ Computed Tomography Department, The First Affiliated Hospital of Harbin \\ Medical University, Harbin, China; ${ }^{4}$ Department of Anesthesiology, The First People's Hospital of Wenling, Taizhou, China \\ Contributions: (I) Conception and design: F Li; (II) Administrative support: K Lin; (III) Provision of study materials or patients: L Cheng; (IV) \\ Collection and assembly of data: L Cheng, X Zhu, Y Liu; (V) Data analysis and interpretation: K Zhu, K Lin; (VI) Manuscript writing: All authors; \\ (VII) Final approval of manuscript: All authors. \\ Correspondence to: Fujun Li. Department of Anesthesiology, The First Affiliated Hospital of Harbin Medical University, No. 23 Postal Street, \\ Nangang District, Harbin 150001, China. Email: lifujun_hydyy@163.com.
}

Background: Acyl-CoA synthetase long chain family member 4 (ACSL4) has been reported to serve as a major player in the progress of ferroptosis in various diseases. Nevertheless, the functional role and mechanism of ACSL4 in sevoflurane (sev)-induced neuronal death has never been elucidated.

Methods: Cell viability was assessed using Cell Counting Kit-8 (CCK-8). Iron levels, reactive oxygen species (ROS) production, and malondialdehyde (MDA), 4-hydroxynonenal (4-HNE), and glutathione (GSH) content were determined to assess ferroptosis level. Quantitative reverse transcription-polymerase chain reaction (qRT-PCR) and western blot were undertaken for the measurement of gene expression.

Results: Sev hindered the viability of SH-SY5Y cells and suppression of ferroptosis by ferrostatin-1 (Fer1) mitigated sev-induced inhibition of SH-SY5Y cell viability. Sev treatment increases the $\mathrm{Fe}^{2+}$ level and decreases the mRNA levels of SLC7A11 and GPX4 in SH-SY5Y cells. Sev increased the expression of ACSL4. Moreover, silencing of ACSL4 could abrogate sev-induced cell damage, as evidenced by increases in cell viability, GPX4 protein levels, and decreases in iron levels, ROS production, and MDA and 4-HNE content. Remarkably, sev hindered the activation of the 5' AMP-activated protein kinase (AMPK)/ mammalian target of rapamycin (mTOR) signaling, which was diminished by knockdown of ACSL4. Moreover, inhibition of the AMPK/mTOR signaling by compound C could mitigate the protective effect of ACSL4 silencing against sev-induced ferroptotic cell death.

Conclusions: Downregulation of ACSL4 restrained sev-induced ferroptotic cell death via AMPK/mTOR signaling, providing the basis for an approach to alleviate sev-induced postoperative cognitive dysfunction (POCD).

Keywords: Acyl-CoA synthetase long chain family member 4 (ACSL4); sevoflurane (sev); ferroptosis; 5' AMPactivated protein kinase/mammalian target of rapamycin (AMPK/mTOR)

Submitted Jul 23, 2021. Accepted for publication Sep 10, 2021.

doi: $10.21037 / \mathrm{atm}-21-4249$

View this article at: https://dx.doi.org/10.21037/atm-21-4249 


\section{Introduction}

Postoperative cognitive dysfunction (POCD) is one of the most common postoperative complications characterized by mental derangement, anxiety, cognitive dysfunction, memory impairment, and loss of attention (1). There is a chance that POCD may develop into severe neuronal diseases, such as dementia (2). It has been noticed by scientists in China and abroad for the last few years, because of its negative influence on hospitalization time, quality of life, and mortality rate (3). However, up to now, the pathophysiological mechanisms of POCD have not been completely clarified. Sevoflurane (sev) is frequently used in clinical anesthesia, and it has the characteristics of short onset, rapid recovery, and better practical control, but it also has some virulence (4). Sev has been described as causing neurotoxicity in several studies, leading to cognitive dysfunction (5). One study demonstrated that sev exposure can reduce glutamatergic neurons in the basolateral amygdala, decrease neural activation and induce adulthood fear induced-learning deficits in neonatal mice (6). Thus, reducing sev-induced neurotoxicity is of significance in the treatment of POCD.

Ferroptosis is a recently recognized non-apoptotic cell death process that involves iron overload and lipid peroxide and reactive oxygen species (ROS) accumulation (7). Accumulating evidence from clinical settings has linked ferroptosis with the development of human diseases, including nervous system diseases (8). A study by $\mathrm{Lu}$ et al. suggested that sev exposure could cause cognitive dysfunction without affecting neuroapoptosis, revealing that sev results in cognitive dysfunction by inducing other cell death mechanisms (9). Remarkably, iron metabolic derangement has been reported to be highly correlated with the pathogenesis of anesthetic drug, including sevinduced neurotoxicity and neurological deficits (10). Studies in rodents have reported that general anesthesia, including ketamine and sev, led to the disruption of iron homeostasis and induced iron overload via divalent metal transporter 1-mediated NMDAR-RASD1 signaling and, hence, resulted in cognitive impairment (11). However, the mechanisms involved in sev-induced ferroptotic neuron death are still to be explored.

Zou et al. demonstrated that differentiated SH-SY5Y cells showed higher sensitivity to GPX4-inhibitioninduced ferroptosis than the parental cells (12). This research suggested that the susceptibility to iron death can change in response to external inducers or stress stimuli. The specific molecular mechanism by which susceptibility to ferroptosis is dynamically regulated remains unclear. The external stimuli-caused abnormal expression of iron death-related genes may be responsible for changes in cell susceptibility to iron death. Acyl-CoA synthetase long chain family member 4 (ACSL4) belongs to the long chain acyl coenzyme A synthase family and is the key enzyme that controls the metabolism of fatty acids (13). It can catalyze the synthesis of acyl-CoA from fatty acids, which is the major process linked with ferroptosis (14). It is recognized as a crucial regulator of ferroptosis, and forced expression of ACSL4 induced the activation of the ferroptosis pathway $(15,16)$. Moreover, silencing of ACSL4 promoted the proliferation of glioma cells by inhibiting ferroptosis (17). However, relatively few studies have elucidated the association between ACSL4 and sev-induced ferroptotic neuronal death. In this study, we aimed to elucidate if ACSL4 participates in sev-induced ferroptotic neuronal death and found that downregulation of ACSL4 restrained sev-induced ferroptotic cell death via 5' AMP-activated protein kinase (AMPK)/mammalian target of rapamycin (mTOR) signaling. Therefore, targeting ACSL 4 might be a potential therapeutic strategy for alleviating sev-induced POCD. We present the following article in accordance with the MDAR reporting checklist (available at https:// dx.doi.org/10.21037/atm-21-4249).

\section{Methods}

\section{Cell culture}

Human neuroblastoma cell line (SH-SY5Y; organism: Homo sapiens) from American type culture collection (catalog number: CRL-2266, Manassas, VA, USA) were grown in Dulbecco's modified Eagle medium: nutrient mixture F-12 (DMEM/F12, ThermoFisher, Waltham, MA, USA) medium plus $10 \%$ fetal bovine serum (FBS) at $37{ }^{\circ} \mathrm{C}$ in $95 \%$ air $/ 5 \% \mathrm{CO}_{2}$.

\section{Cell transfection}

Both si-ACSL4 and si-NC were acquired from GenePharma (Shanghai, China) and transfected into SH-SY5Y cells in the presence of Lipofectamine 2000 (ThermoFisher, Waltham, MA, USA), following the product manual. Afterward, SHSY5Y cells were exposed to $4.1 \%$ sev (Sigma-Aldrich, St. Louis, MO, USA) for $6 \mathrm{~h}$. 


\section{Drug treatment}

To evaluate the cytotoxic effect of sev, SH-SY5Y cells were stimulated with $4.1 \%$ sev for different durations $(0,2,4,6$, or $12 \mathrm{~h}$ ).

To confirm if sev induces neuronal cell death via ferroptosis, SH-SY5Y cells were treated with ferrostatin-1 (Fer-1; $10 \mu \mathrm{M}$; a ferroptosis inhibitor) from SigmaAldrich, (St. Louis, MO, USA) or chelating agent [20 $\mu \mathrm{M}$; deferoxamine mesylate (DFOM)] from Sigma-Aldrich (St. Louis, MO, USA) for $12 \mathrm{~h}$, followed by incubation with $4.1 \%$ sev for $6 \mathrm{~h}$.

To verify whether ACSL4 contributes to sev-induced ferroptosis via AMPK/mTOR signaling, SH-SY5Y cells were cultured in DMEM/F12 medium containing compound $\mathrm{C}(10 \mu \mathrm{M})$ for $12 \mathrm{~h}$ after si-ACSL4 or si-NC transfection, and then exposed to $4.1 \%$ sev for $6 \mathrm{~h}$.

\section{Cell Counting Kit-8 (CCK-8) assay}

CCK-8 (Solarbio, Beijing, China) was utilized to determine the viability of SH-SY5Y cells. After sev exposure, SHSY5Y cells were cultured in DMEM/F12 medium plus $10 \mu \mathrm{L}$ CCK-8 solution at $37{ }^{\circ} \mathrm{C}$ in $95 \%$ air $/ 5 \% \mathrm{CO}_{2}$ for $4 \mathrm{~h}$, and then tested for absorbance at $450 \mathrm{~nm}$ using a microplate reader.

\section{Measurement of iron, malondialdehyde (MDA), 4-hydroxynonenal (4-HNE), and glutatbione (GSH) levels}

The levels of MDA, 4-HNE, and GSH were assessed using the Lipid Peroxidation (MDA) Assay Kit (SigmaAldrich, St. Louis, MO, USA), Lipid Peroxidation (4-HNE) Assay Kit (Abcam, Cambridge, MA, USA), GSH Assay Kit (colorimetric; Abcam, Cambridge, MA, USA), and Iron Assay Kit (Sigma-Aldrich, St. Louis, MO, USA) following the directions of the manufacturer.

\section{Detection of ROS level}

ROS was detected by staining the SH-SY5Y cells using a commercial dichlorofluorescin diacetate (DCFDA) Cellular ROS Assay Kit (Abcam, Cambridge, MA, USA) following protocols supplied by the manufacturer. Cells were seeded and allow attachment of adherent cells in 96-well plate. After washing once with buffer (provided in the kit), $\mathrm{SH}$ SY5Y cells were stained with DCFDA for $45 \mathrm{~min}$ at $37{ }^{\circ} \mathrm{C}$ in dark conditions. After washing once with buffer,
ROS generation was analyzed with a microplate reader or fluorescent microscope. Photos were taken of three randomly selected fields of view.

\section{Quantitative reverse transcription-polymerase chain reaction (qRT-PCR) assay}

Using a TRIzol RNA isolation kit from Life Technologies (Rockville, MD, USA), RNA isolation was undertaken following the manufacturer's recommendations. A qRTPCR assay was carried out using the PrimeScript RT Reagent Kit (TaKaRa, Dalian, China) on a QX100 Droplet Digital PCR system (Bio-Rad, Herkules, CA, USA). The relative expression of ACSL4 was normalized to that of $G A P D H$, according to the $2^{-\triangle \Delta \mathrm{Ct}}$ method. Special primers used in our experiments were as follows: ACSL4 forward: 5'-CATCCCTGGAGCAGATACTCT-3', and reverse: 5'-TCACTTAGGATTTCCCTGGTCC-3'; GPX4, forward: 5'-GGGCTACAACGTCAAATTCG-3', and reverse: 5'-TCCACTTGATGGCATTTCCC-3'; SLC7A11 forward: 5' - TGCTGGGCTGATT TTATCTTCG-3'; reverse: 5'-GAAAGGGCAACCAT GAAGAGG-3'; GAPDH, 5'-GGAGCGAGATCCC TCCAAAAT-3', and reverse: 5'-GGCTGTTGTCATAC TTCTCATGG-3'.

\section{Western blot}

Total protein was isolated from SH-SY5Y cells with the help of ice-cold radioimmunoprecipitation assay buffer containing protease and phosphatase inhibitors, followed by quantification with the BCA Protein Assay Kit from Abcam (Cambridge, MA, USA) following the manual. Protein separation was conducted using $12 \%$ sodium dodecyl sulfate-polyacrylamide gel electrophoresis, and then the proteins were electrotransferred onto the polyvinylidenefluoride membrane. After blockade with $5 \%$ fat-free milk, the membrane was incubated with the primary antibodies at $4{ }^{\circ} \mathrm{C}$ overnight and then probed with horseradish peroxidase-conjugated secondary antibodies for $1 \mathrm{~h}$ at room temperature before visualization using High Sensitivity ECL Substrate Kit (Abcam, Cambridge, MA, USA). Primary antibodies in our study included those for ACSL4 (catalogue number: NB300-861; Novus, Shanghai, China), GPX4 (catalogue number: NBP2-76933; Novus, Shanghai, China), AMPK (catalogue number: MA515815; ThermoFisher Scientific, Waltham, MA, USA), phosphorylated AMPK (pAMPK; catalogue number: 44- 

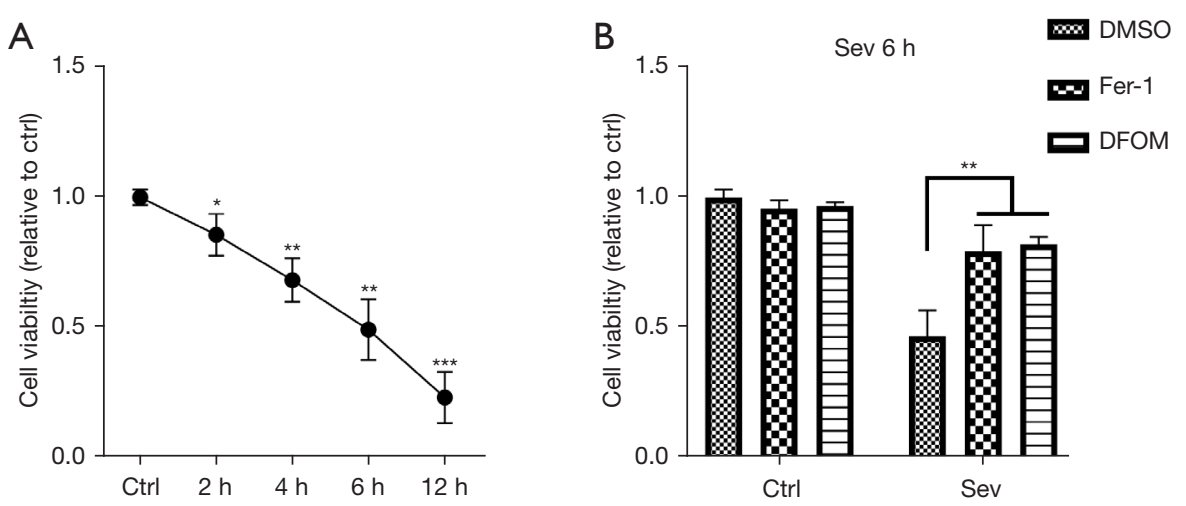

Figure 1 Sev effectively induced ferroptosis in SH-SY5Y cells. SH-SY5Y cells were exposed to 4.1\% sev for different durations (0, 2, 4, 6, or 12 h). (A) CCK-8 assay was performed to detect the viability of SH-SY5Y cells in different groups. (B) SH-SY5Y cells were treated with Fer1 and DFOM for $12 \mathrm{~h}$ and then exposed to $4.1 \%$ sev for $6 \mathrm{~h}$, and then subjected to the CCK-8 assay. ${ }^{*}, \mathrm{P}<0.05 ;{ }^{* *}, \mathrm{P}<0.01 ;{ }^{* * *}, \mathrm{P}<0.001$. Sev, sevoflurane; CCK-8, Cell Counting Kit-8; Fer-1, ferrostatin-1; DFOM, deferoxamine mesylate.

1150G; ThermoFisher Scientific, Waltham, MA, USA), mTOR (catalogue number: PA5-34663; ThermoFisher Scientific, Waltham, MA, USA), and phosphorylated mTOR (p-mTOR; catalogue number: 44-1125G; ThermoFisher Scientific, Waltham, MA, USA) using GAPDH (catalogue number: NB100-56875; Novus, Vancouver, Canada) as an internal control.

\section{Statistical analysis}

Experimental data were analyzed by investigators who were blind to grouping and treatment. All the experiments were repeated independently three times $(\mathrm{n}=3$ biological replicates). Our results are reported as the mean \pm standard deviation using Graphpad Prism (Graph Pad, San Diego, CA, USA) and analyzed by Student's $t$-test (comparison between two groups) or one-way analysis of variance (comparison between multiple groups) using SPSS version 18.0. The significance threshold was set at $\mathrm{P}<0.05$ for all the tests.

\section{Results}

\section{Sev effectively induced ferroptosis in SH-SY5Y cells}

As evaluated by CCK-8 assay, sev management effectively reduced the viability of SH-SY5Y cells in a time-dependent manner (Figure 1A). It is worth noting that Fer-1 and DFOM could restrain $4.1 \%$ sev-induced decline of SHSY5Y cell viability (Figure $1 B$ ). These results suggest that sev-induced neuronal damage in SH-SY5Y cell was mediated by ferroptosis.

\section{Sev treatment increases the $\mathrm{Fe}^{2+}$ level and decreases the mRNA levels of SLC7A11 and GPX4}

We detected $\mathrm{Fe}^{2+}$ level and the expression of iron deathrelated genes (SLC7A11 and GPX4) in SH-SY5Y cells at 6, 12,24 , and $48 \mathrm{~h}$ after sev treatment. Results showed that sev-treated SH-SY5Y cells exhibited higher $\mathrm{Fe}^{2+}$ level at $6,12,24$, and $48 \mathrm{~h}$ after sev treatment than control cells without sev treatment (Figure 2A). After sev treatment, the mRNA levels of SLC7A11 and GPX4 in SH-SY5Y cells decreased gradually over time (Figure 2B,2C).

\section{Downregulation of ACSL4 mitigated sev-induced cell injury in SH-SY $5 Y$ cells}

To explore the impact of sev on ACSL4 expression, SHSY5Y cells were exposed to $4.1 \%$ of sev. Results showed that sev-induced elevation of ACSL4 expression was blocked in SH-SY5Y cells after si-ACSL4 transfection relative to the sev + si-ACSL4 group (Figure $3 A$ ). In line with this, knockdown of ACSL4 strikingly abrogated sev-induced inhibition of SH-SY5Y cell viability in the CCK-8 assay (Figure 3B).

\section{Downregulation of ACSL4 inbibited sev-induced ferroptosis}

To investigate the role of ACSL4 in sev-induced ferroptosis, we knocked down ACSL4 in SH-SY5Y cells using si-ACSL4 

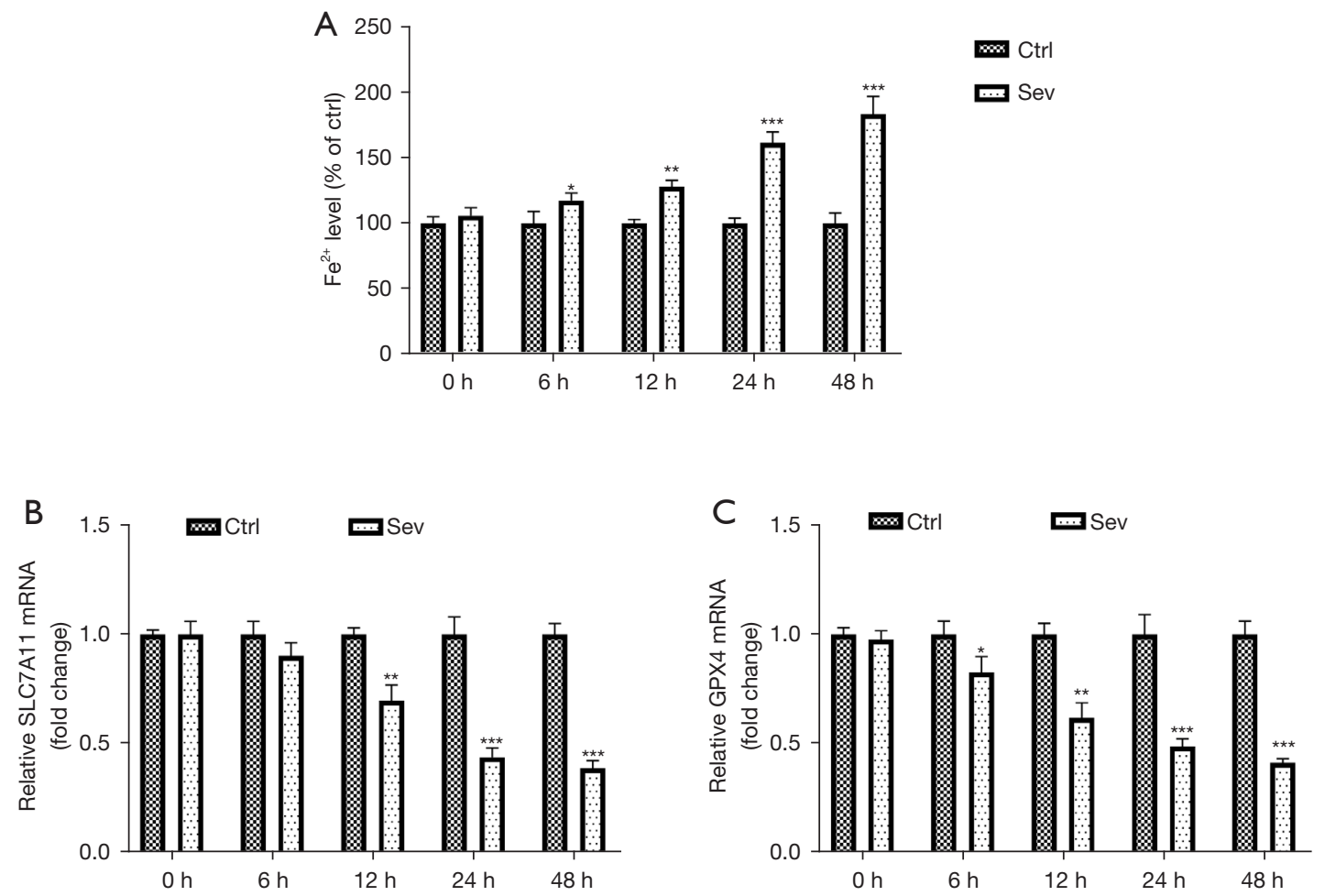

Figure $2 \mathrm{Sev}$ treatment increases the $\mathrm{Fe}^{2+}$ level and decreases the mRNA levels of SLC7A11 and GPX4. (A) Fe ${ }^{2+}$ level in SH-SY5Y cells was detected at 6, 12, 24, and $48 \mathrm{~h}$ after sev treatment. qRT-PCR was conducted to detected the mRNA levels of SLC7A11 (B) and GPX4 (C). *, $\mathrm{P}<0.05$; **, $\mathrm{P}<0.01$; ${ }^{* *}, \mathrm{P}<0.001$. Sev, sevoflurane; $\mathrm{Fe}^{2+}$, ferrous ion; qRT-PCR, quantitative reverse transcription-polymerase chain reaction.
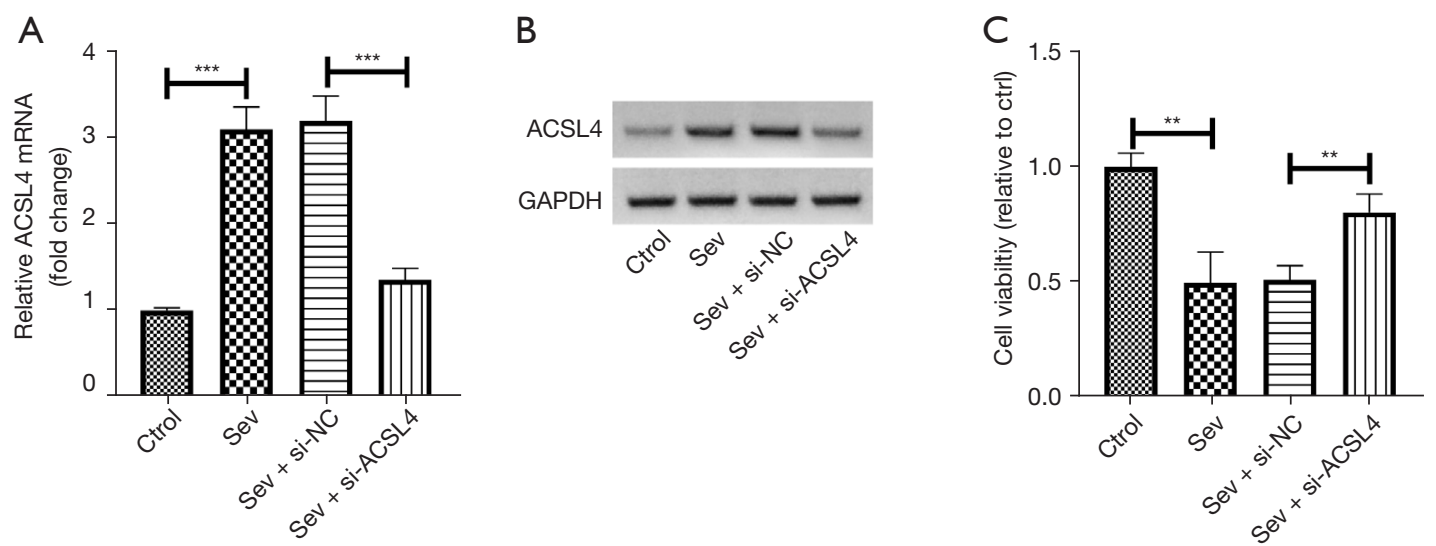

Figure 3 Downregulation of ACSL4-mitigated sev-induced cell injury in SH-SY5Y cells. (A) si-ACSL4 or si-NC was transfected into SHSY5Y cells and incubated for $24 \mathrm{~h}$. Following treatment with $4.1 \%$ sev for $6 \mathrm{~h}, \mathrm{SH}-\mathrm{SY} 5 \mathrm{Y}$ cells were subjected to qRT-PCR and western blot assays. (B) Cell viability was also examined using a CCK-8 assay. **, $\mathrm{P}<0.01$; ***, $\mathrm{P}<0.001$. ACSL4, acyl-CoA synthetase long chain family member 4; sev, sevoflurane; qRT-PCR, quantitative reverse transcription-polymerase chain reaction; CCK-8, Cell Counting Kit-8. 

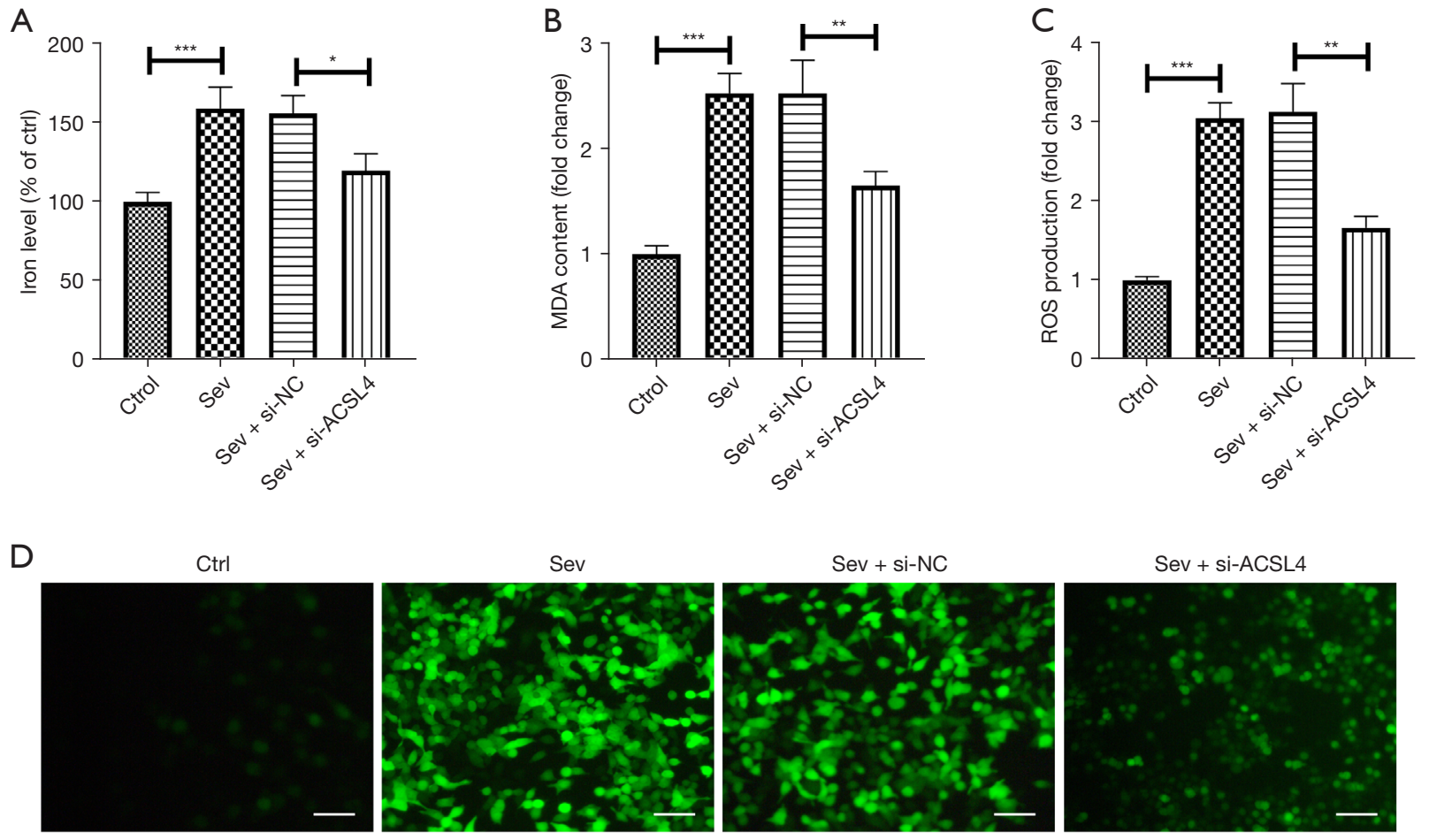

E

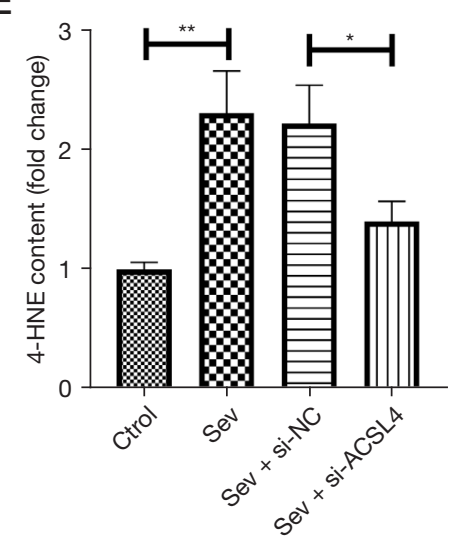

$\mathrm{F}$

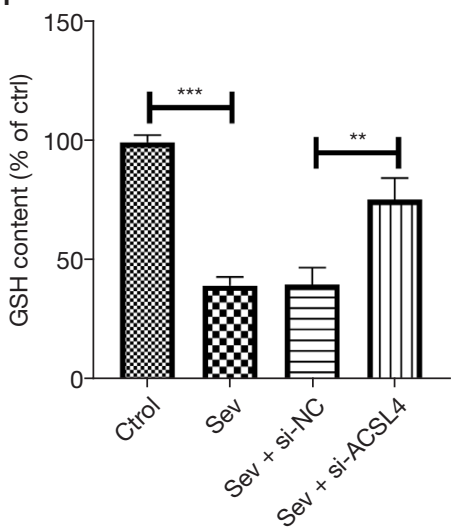

G

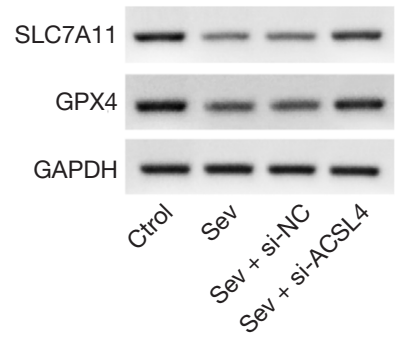

Figure 4 Downregulation of ACSL4 inhibited sev-induced ferroptosis. si-ACSL4 or si-NC was transfected into SH-SY5Y cells and incubated for $24 \mathrm{~h}$, followed by treatment with $4.1 \% \mathrm{sev}$ for $6 \mathrm{~h}$. Commercial kits were utilized to detect iron level (A) and contents of MDA (B), ROS (C,D; DCFDA staining assay), 4-HNE (E) and GSH (F). (G) Western blot analysis of SLC7A11 and GPX4 protein expression in SH-SY5Y cells. Scale bar $=50 \mu \mathrm{m} .{ }^{*}, \mathrm{P}<0.05 ;{ }^{* *}, \mathrm{P}<0.01$; ${ }^{* * *}, \mathrm{P}<0.001$. ACSL4, acyl-CoA synthetase long chain family member 4; sev, sevoflurane; MDA, malondialdehyde; ROS, reactive oxygen species; 4-HNE, 4-hydroxynonenal; GSH, glutathione.

and then determined the changes of the ferroptotic indexes. It was found that sev treatment caused the increase of iron levels, which was abolished by downregulation of ACSL4 (Figure 4A). Also, sev increased the content of MDA and 4-HNE and the production of ROS, and these increases were diminished following si-ACSL4 transfection (Figure
$4 B-4 E)$. Furthermore, an elevation of GSH content was discovered in SH-SY5Y cells stimulated with sev, whereas sev-induced increase of GSH content was blocked by silencing of ACSL4 (Figure $4 F$ ). Moreover, the protein levels of SLC7A11 and GPX4 were reduced following sev administration, and the reduction of SLC7A11 and GPX4 
A

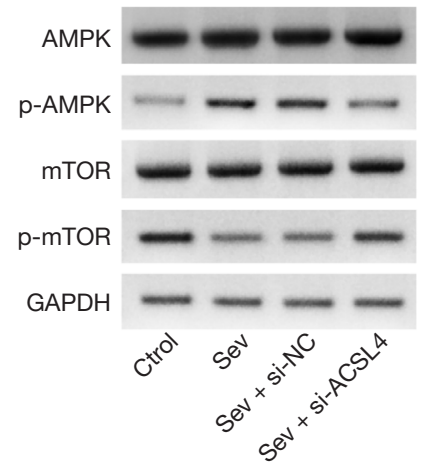

B

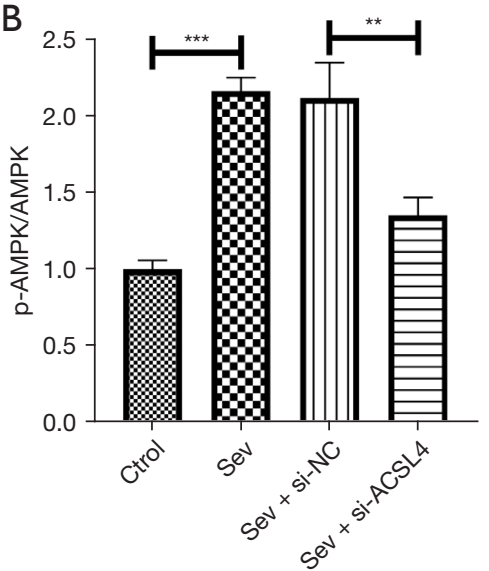

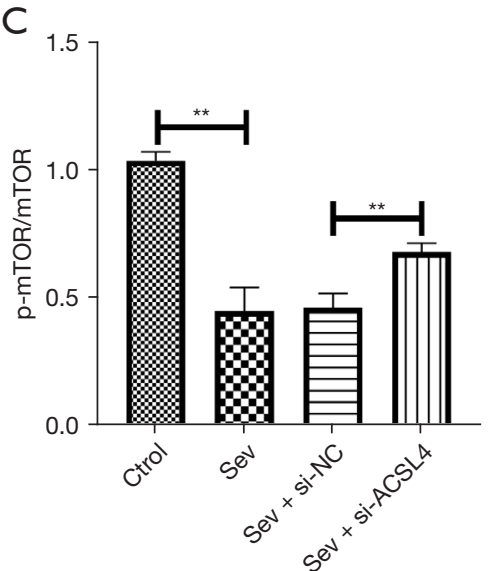

Figure 5 ACSL4 regulated the AMPK/mTOR pathway in sev-stimulated SH-SY5Y cells. SH-SY5Y cells were transfected with si-ACSL4 or si-NC for $24 \mathrm{~h}$ and treated with $4.1 \%$ sev for $6 \mathrm{~h}$, and then tested for the expression of AMPK, pAMPK, mTOR, and p-mTOR using western blot. (A) Representative images for western blot of AMPK, pAMPK, mTOR, and p-mTOR expression in SH-SY5Y cells are shown. Quantification of (B) p-AMPK/AMPK and (C) p-mTOR/mTOR ratios in SH-SY5Y cells. **, $\mathrm{P}<0.01$; *** $\mathrm{P}<0.001$. ACSL4, acyl-CoA synthetase long chain family member 4; AMPK, 5' AMP-activated protein kinase; mTOR, mammalian target of rapamycin; sev, sevoflurane; pAMPK, phosphorylated AMPK; p-mTOR, phosphorylated mTOR; sev, sevoflurane.

expression induced by sev was mitigated after knockdown of ACSL4 (Figure 4G).

\section{ACSL4 regulated the AMPK/mTOR patbway in sev- stimulated SH-SYSY cells}

To verify if the impact of ACSL4 on sev-induced ferroptosis is mediated by AMPK/mTOR signaling, SH-SY5Y cells were transfected with si-ACSL4 or si-NC for $24 \mathrm{~h}$ and treated with $4.1 \%$ sev for $6 \mathrm{~h}$. Subsequently, the expression levels of AMPK, pAMPK, mTOR, and p-mTOR were tested using western blot. It was found that sev restrained the activation of AMPK/mTOR signaling with the increased expression of $\mathrm{p}-\mathrm{AMPK}$ and decreases in $\mathrm{p}-\mathrm{mTOR}$ protein levels, which was reversed following silencing of ACSL4 (Figure 5A). Similarly, sev increased the ratios of $\mathrm{p}$-AMPK/AMPK in SH-SY5Y cells, and this increase was abolished after downregulation of ACSL4 (Figure 5B). On the contrary, sev caused a decrease in $\mathrm{p}-\mathrm{mTOR} / \mathrm{mTOR}$ ratio, which was abrogated when SH-SY5Y cells were transfected with si-ACSL4 (Figure 5B,5C).

\section{ACSL4 knockdown attenuated sev-induced ferroptosis $S H$ - SYSY cells through the AMPK/mTOR patbway}

To further verify if ACSL4 controls sev-induced ferroptosis via the AMPK/mTOR signaling, SH-SY5Y cells were transfected with si-ACSL4 for $12 \mathrm{~h}$, treated with compound $\mathrm{C}$ for $12 \mathrm{~h}$, and then incubated for $6 \mathrm{~h}$ in the medium plus $4.1 \%$ sev. As expected, compound $\mathrm{C}$ hindered the effect of si-ACSL4 on sev-induced inhibition of cell viability in SH-SY5Y cells (Figure 6A). In addition, deletion of ACSL4 mitigated sev-induced elevation of iron levels, which was abrogated by compound $\mathrm{C}$ management (Figure $6 B$ ). The elevations of MDA and 4-HNE content and ROS generation induced by sev was inhibited by ACSL4 knockdown, and this inhibition was diminished by compound $\mathrm{C}$ treatment (Figure $6 C-6 F$ ). Moreover, compound $\mathrm{C}$ treatment blocked ACSL4 silencing-induced inhibition of GSH content in sev-stimulated SH-SY5Y cells (Figure 6G). Moreover, compound C management could eliminate the effect of ACSL4 knockdown on sev-caused reduction of GPX expression in SH-SY5Y cells (Figure 6H).

\section{Discussion}

There is a growing concern that anesthetic drugs exert a toxic action on neurons and result in persistent cognitive impairment $(18,19)$. Nevertheless, to date, their neurotoxic action has not yet been clarified. In the last few years, a link has been demonstrated between anesthetic druginduced neurotoxicity and cognitive deficits and cell death mechanisms, especially ferroptosis. It was found that the massive accumulation of iron can trigger neurotoxicity 

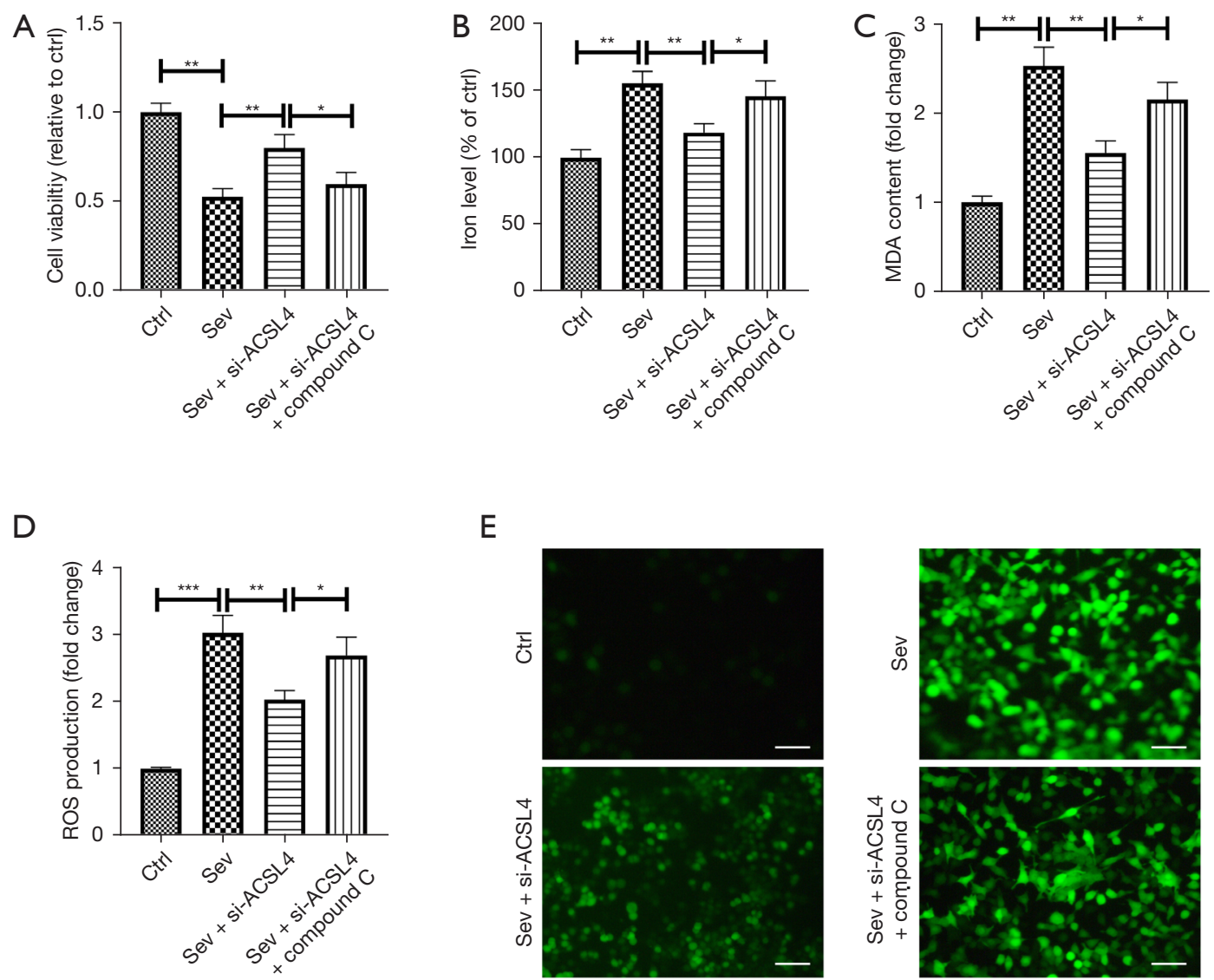

\section{E}
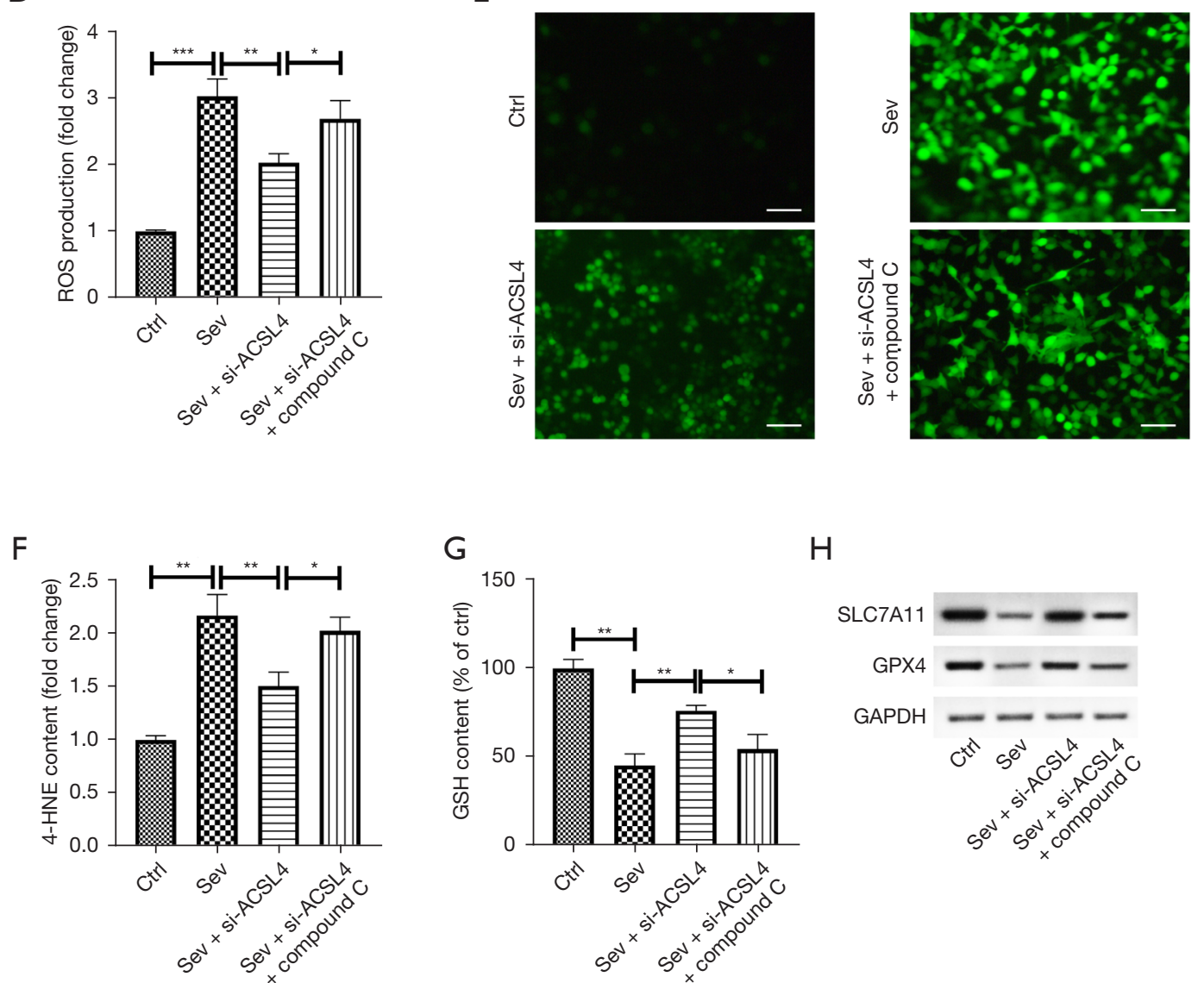

$\mathrm{H}$

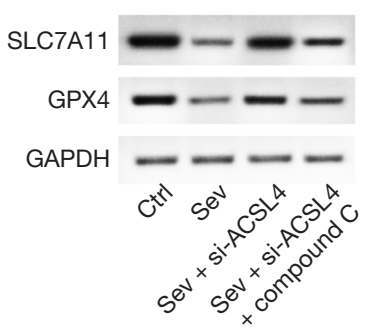

Figure 6 ACSL4 knockdown attenuated sev-induced ferroptosis SH-SY5Y cells through the AMPK/mTOR pathway. SH-SY5Y cells were transfected with si-ACSL4 for $12 \mathrm{~h}$, treated with compound $\mathrm{C}$ for $12 \mathrm{~h}$ and then incubated in the medium plus $4.1 \%$ sev for $6 \mathrm{~h}$. (A) Cell viability was analyzed using the CCK-8 assay. Commercial kits were utilized to detect the levels of iron (B), MDA (C), ROS (D,E; DCFDA staining assay), 4-HNE (F), and GSH $(\mathrm{G})$. (H) Western blot analysis of GPX4 protein expression in SH-SY5Y cells. Scale bar $=50 \mu \mathrm{m} .{ }^{*}$, $\mathrm{P}<0.05 ;{ }^{* *}, \mathrm{P}<0.01 ;{ }^{* *}, \mathrm{P}<0.001$. ACSL4, acyl-CoA synthetase long chain family member 4; sev, sevoflurane; AMPK, 5 ' AMP-activated protein kinase; mTOR, mammalian target of rapamycin; CCK-8, Cell Counting Kit-8; MDA, malondialdehyde; ROS, reactive oxygen species; 4-HNE, 4-hydroxynonenal; GSH, glutathione. 
and severe cognitive dysfunction (20). There is evidence from an in vitro study that isoflurane administration could restrain the expression of GPX4, induce the production of ROS and disrupt mitochondrial membrane potential, finally leading to neuronal death. Moreover, Fer-1 pretreatment could mitigate the toxic effect of isoflurane on mouse primary cortical neurons (21). Additionally, treatment of SH-SY5Y cells with isoflurane caused the phosphorylation of beclin 1 and promoted the formation of beclin 1-solute carrier family 7 member 11 complex, thereby aggravating isoflurane-induced ferroptotic neuronal death (22). This aforementioned evidence indicates that ferroptosis is a major mechanism of anesthetic drug-induced neurotoxicity. Thus, reducing anesthetic drug-induced ferroptotic neuronal death is a promising therapeutic strategy for alleviating anesthetic drug-induced POCD. In the current study, we found that sev dose time-dependently hindered the viability of SH-SY5Y cells. Moreover, Fer-1 and DFOM could restrain $4.1 \%$ sev-induced decline of cell viability in SH-SY5Y cells, revealing that sev can induce ferroptosis in SH-SY5Y cells, and inhibition of ferroptosis can protect against sev-induced neuronal damage.

To date, the significant role of ACSL4 in ferroptosis has been well established (23). Recently, several lines of evidence suggested that ACSL4 takes part in the regulation of ferroptosis in various human diseases (24). Previously, Ma et al. found that upregulation of ACSL4 increased the sensitivity of ovarian cancer cells to erastin- and RSL3induced ferroptosis (25). Xu et al. stated that knockdown of ACSL4 reduced lipid peroxidation, increased the expression of GSH and GPX4, and hence restrained ferroptosis, ultimately attenuating ischemia reperfusion-induced lung injury (26). Notably, ACSL4 has been shown to be upregulated in the brain tissues of rats with subarachnoid hemorrhage. Furthermore, both silencing of ACSL4 or inhibition of ferroptosis could mitigate subarachnoid hemorrhage-induced neuronal injury, indicating that ACSL4 exacerbates subarachnoid hemorrhage-induced early brain injury via ferroptosis (27). However, although the clinical effect of ACSL4 strikingly promotes ferroptosis in various diseases, its effects in sev-induced neurotoxicity remain elusive. In this work, sev increased the expression of ACSL4. Moreover, silencing of ACSL4 could abrogate sevinduced neuronal cell death, as evidenced by the increases in cell viability, GSH content, and GPX4 protein levels, and the decreases in iron levels, ROS production, MDA content, and 4-HNE content, thus demonstrating the vital role of ACSL4 in sev-induced ferroptotic neuronal death. MicroRNAs regulates the expression of downstream target genes at the post-transcriptional level. miR-212-5p attenuates ferroptotic neuronal death in a mouse model of traumatic brain injury by downregulating prostaglandinendoperoxide synthase-2 expression (28). It remains unclear whether Sev-caused upregulation of ACSL4 in SH-SY5Y cells is due to changes in miRNAs, which needs to be further studied.

Although evidence on the role of ACSL4 in sevinduced ferroptotic neuronal death is available, the signaling mechanism of ACSL4 action remains unknown. As a central energy metabolic switch, AMPK plays a vital role in the process of cellular function, such as cell proliferation, death, and survival (29). It is essential for maintaining mitochondrial homeostasis, which serves as a momentous player in controlling ferroptosis $(30,31)$. Notably, AMPK could inhibit acetyl-CoA carboxylasemediated polyunsaturated fatty acid biosynthesis, leading to ferroptosis repression (32). Similarly, the benzopyran derivative 2-imino-6-methoxy-2H-chromene-3carbothioamide induced ROS-induced ferroptosis by repressing the activation of AMPK/mTOR signaling in colorectal cancer cells, revealing that the activation of AMPK/mTOR signaling suppresses ferroptosis (33). Conversely, a recent study also showed that SIRT3 deficiency hindered the activation of AMPK/mTOR signaling and increased the expression of GPX4, thereby suppressing high glucose- and erastin-induced autophagydependent ferroptosis in trophoblastic cells (34). Moreover, inhibition of AMPK could impair SIRT3-enhanced ferroptosis, implying that the activation of AMPK/mTOR signaling induces ferroptosis (34). In contrast to the previous study, our research manifested that sev hindered the activation of the AMPK/mTOR signaling, which was diminished by knockdown of ACSL4. Importantly, inhibition of AMPK/mTOR signaling by compound $\mathrm{C}$ could mitigate the protective effect of ACSL4 silencing against sevinduced ferroptotic cell death. The aforementioned findings revealed that knockdown of ACSL4 exhibited its protective effect against sev-induced ferroptosis by activating AMPK/ mTOR signaling.

Cognitive dysfunction is also one of the most representative clinical features in neurodegenerative disorders, for example, Alzheimer's disease (AD) and Parkinson's disease (PD) (35). In recent years, several studies have demonstrated the involvement of ferroptosis in the pathogenesis of neurodegenerative disorders $(36,37)$. It is inconclusive whether the mechanism of ACSL4-mediated 
ferroptosis is effective in the experimental models of PD and AD. As a shortcoming of this study, the results of the in vitro experiments were not confirmed in an in vivo model. Further research should be done to verify the functional role of ACSL4 in a mouse model of sev-induced neuronal damage.

In summary, we found that silencing of ACSL4 protected SH-SY5Y cells against sev-induced ferroptosis via AMPK/ mTOR signaling. Our data has theoretical significance and clinical value and provides a potential therapy for sevinduced POCD.

\section{Acknowledgments}

Funding: None.

\section{Footnote}

Reporting Checklist: The authors have completed the MDAR reporting checklist. Available at https://dx.doi. org/10.21037/atm-21-4249

Data Sharing Statement: Available at https://dx.doi. org/10.21037/atm-21-4249

Conflicts of Interest: All authors have completed the ICMJE uniform disclosure form (available at https://dx.doi. org/10.21037/atm-21-4249). The authors have no conflicts of interest to declare.

Ethical Statement: The authors are accountable for all aspects of the work in ensuring that questions related to the accuracy or integrity of any part of the work are appropriately investigated and resolved.

Open Access Statement: This is an Open Access article distributed in accordance with the Creative Commons Attribution-NonCommercial-NoDerivs 4.0 International License (CC BY-NC-ND 4.0), which permits the noncommercial replication and distribution of the article with the strict proviso that no changes or edits are made and the original work is properly cited (including links to both the formal publication through the relevant DOI and the license). See: https://creativecommons.org/licenses/by-nc-nd/4.0/.

\section{References}

1. Nathan N. Beyond emergence: understanding postoperative cognitive dysfunction (POCD). Anesth Analg 2018;127:323.

2. Needham MJ, Webb CE, Bryden DC. Postoperative cognitive dysfunction and dementia: what we need to know and do. Br J Anaesth 2017;119:i115-25.

3. Santarpino G, Moscarelli M. Postoperative cognitive dysfunction: a forgotten part of the quality of life? Ann Thorac Surg 2019;108:1583.

4. Belrose JC, Noppens RR. Anesthesiology and cognitive impairment: a narrative review of current clinical literature. BMC Anesthesiol 2019;19:241.

5. Chen Y, Zhang P, Lin X, et al. Mitophagy impairment is involved in sevoflurane-induced cognitive dysfunction in aged rats. Aging (Albany NY) 2020. [Epub ahead of print]. doi: 10.18632/aging.103673.

6. Satomoto M, Sun Z, Adachi YU, et al. Neonatal sevoflurane exposure induces adulthood fear-induced learning disability and decreases glutamatergic neurons in the basolateral amygdala. J Neurosurg Anesthesiol 2018;30:59-64.

7. Hirschhorn T, Stockwell BR. The development of the concept of ferroptosis. Free Radic Biol Med 2019;133:130-43.

8. Wu Y, Song J, Wang Y, et al. The potential role of ferroptosis in neonatal brain injury. Front Neurosci 2019;13:115.

9. Lu Y, Huang Y, Jiang J, et al. Neuronal apoptosis may not contribute to the long-term cognitive dysfunction induced by a brief exposure to $2 \%$ sevoflurane in developing rats. Biomed Pharmacother 2016;78:322-8.

10. Zuo Y, Chang Y, Thirupathi A, et al. Prenatal sevoflurane exposure: effects of iron metabolic dysfunction on offspring cognition and potential mechanism. Int J Dev Neurosci 2021;81:1-9.

11. Wu J, Yang JJ, Cao Y, et al. Iron overload contributes to general anaesthesia-induced neurotoxicity and cognitive deficits. J Neuroinflammation 2020;17:110.

12. Zou Y, Henry WS, Ricq EL, et al. Plasticity of ether lipids promotes ferroptosis susceptibility and evasion. Nature 2020;585:603-8.

13. Kuwata H, Hara S. Role of acyl-CoA synthetase ACSL4 in arachidonic acid metabolism. Prostaglandins Other Lipid Mediat 2019;144:106363.

14. Doll S, Proneth B, Tyurina YY, et al. ACSL4 dictates ferroptosis sensitivity by shaping cellular lipid composition. Nat Chem Biol 2017;13:91-8.

15. Yuan $\mathrm{H}, \mathrm{Li} \mathrm{X}$, Zhang X, et al. Identification of ACSL4 as a biomarker and contributor of ferroptosis. Biochem 
Biophys Res Commun 2016;478:1338-43.

16. Kagan VE, Mao G, Qu F, et al. Oxidized arachidonic and adrenic PEs navigate cells to ferroptosis. Nat Chem Biol 2017;13:81-90.

17. Cheng J, Fan YQ, Liu BH, et al. ACSL4 suppresses glioma cells proliferation via activating ferroptosis. Oncol Rep 2020;43:147-58.

18. Verlinde M, Hollmann MW, Stevens MF, et al. Local anesthetic-induced neurotoxicity. Int J Mol Sci 2016;17:339.

19. Ho AM, Fleming ML, Mizubuti GB. Anesthetic neurotoxicity and the developing brain. CMAJ 2017;189:E1028-9.

20. Masaldan S, Bush AI, Devos D, et al. Striking while the iron is hot: iron metabolism and ferroptosis in neurodegeneration. Free Radic Biol Med 2019;133:221-33.

21. Xia Y, Sun X, Luo Y, et al. Ferroptosis contributes to isoflurane neurotoxicity. Front Mol Neurosci 2019;11:486.

22. Liu R, Li X, Zhao G. Beclin1-mediated ferroptosis activation is associated with isoflurane-induced toxicity in SH-SY5Y neuroblastoma cells. Acta Biochim Biophys Sin (Shanghai) 2019;51:1134-41.

23. Kebaili K, Bertrand Y, Foray P, et al. A rare cause of hyponatremia during introductory treatment of acute lymphoblastic leukemia in an infant: inappropriate secretion of atrial natriuretic factor?. Arch Pediatr 1994;1:898-902.

24. Li Y, Feng D, Wang Z, et al. Ischemia-induced ACSL4 activation contributes to ferroptosis-mediated tissue injury in intestinal ischemia/reperfusion. Cell Death Differ 2019;26:2284-99.

25. Ma LL, Liang L, Zhou D, et al. Tumor suppressor miR424-5p abrogates ferroptosis in ovarian cancer through targeting ACSL4. Neoplasma 2021;68:165-73.

26. Xu Y, Li X, Cheng Y, et al. Inhibition of ACSL4 attenuates ferroptotic damage after pulmonary ischemia-reperfusion. FASEB J 2020;34:16262-75.

Cite this article as: Cheng L, Zhu X, Liu Y, Zhu K, Lin K, Li F. ACSL4 contributes to sevoflurane-induced ferroptotic neuronal death in SH-SY5Y cells via the 5' AMP-activated protein kinase/mammalian target of rapamycin pathway. Ann Transl Med 2021;9(18):1454. doi: 10.21037/atm-21-4249
27. Qu XF, Liang TY, Wu DG, et al. Acyl-CoA synthetase long chain family member 4 plays detrimental role in early brain injury after subarachnoid hemorrhage in rats by inducing ferroptosis. CNS Neurosci Ther 2021;27:449-63.

28. Xiao X, Jiang Y, Liang W, et al. miR-212-5p attenuates ferroptotic neuronal death after traumatic brain injury by targeting Ptgs2. Mol Brain 2019;12:78.

29. Lin SC, Hardie DG. AMPK: sensing glucose as well as cellular energy status. Cell Metab 2018;27:299-313.

30. Herzig S, Shaw RJ. AMPK: guardian of metabolism and mitochondrial homeostasis. Nat Rev Mol Cell Biol 2018;19:121-35.

31. Gao M, Yi J, Zhu J, et al. Role of mitochondria in ferroptosis. Mol Cell 2019;73:354-63.e3.

32. Lee H, Zandkarimi F, Zhang Y, et al. Energy-stressmediated AMPK activation inhibits ferroptosis. Nat Cell Biol 2020;22:225-34.

33. Wang DW, Zheng HZ, Cha N, et al. Down-regulation of AHNAK2 inhibits cell proliferation, migration and invasion through inactivating the MAPK pathway in lung adenocarcinoma. Technol Cancer Res Treat 2020;19:1533033820957006.

34. Han D, Jiang L, Gu X, et al. SIRT3 deficiency is resistant to autophagy-dependent ferroptosis by inhibiting the AMPK/mTOR pathway and promoting GPX4 levels. J Cell Physiol 2020;235:8839-51.

35. Xu Y, Yan J, Zhou P, et al. Neurotransmitter receptors and cognitive dysfunction in Alzheimer's disease and Parkinson's disease. Prog Neurobiol 2012;97:1-13.

36. Do Van B, Gouel F, Jonneaux A, et al. Ferroptosis, a newly characterized form of cell death in Parkinson's disease that is regulated by PKC. Neurobiol Dis 2016;94:169-78.

37. Ashraf A, So PW. Spotlight on ferroptosis: iron-dependent cell death in Alzheimer's disease. Front Aging Neurosci 2020;12:196.

(English Language Editor: B. Meiser) 